\title{
Modelling the surface and subsurface Martian radiation environment: Implications for astrobiology
}

\author{
L. R. Dartnell, ${ }^{1}$ L. Desorgher, ${ }^{2}$ J. M. Ward, ${ }^{3}$ and A. J. Coates ${ }^{4}$ \\ Received 7 July 2006; revised 25 September 2006; accepted 22 November 2006; published 30 January 2007.
}

[1] The damaging effect of ionising radiation on cellular structure is one of the prime limiting factors on the survival of life in potential astrobiological habitats. Here we model the propagation of solar energetic protons and galactic cosmic ray particles through the Martian atmosphere and three different surface scenarios: dry regolith, water ice, and regolith with layered permafrost. Particle energy spectra and absorbed radiation dose are determined for the surface and at regular depths underground, allowing the calculation of microbial survival times. Bacteria or spores held dormant by freezing conditions cannot metabolise and become inactivated by accumulating radiation damage. We find that at $2 \mathrm{~m}$ depth, the reach of the ExoMars drill, a population of radioresistant cells would need to have reanimated within the last 450,000 years to still be viable. Recovery of viable cells cryopreserved within the putative Cerberus pack-ice requires a drill depth of at least $7.5 \mathrm{~m}$. Citation: Dartnell, L. R., L. Desorgher, J. M. Ward, and A. J. Coates (2007), Modelling the surface and subsurface Martian radiation environment: Implications for astrobiology, Geophys. Res. Lett., 34, L02207, doi:10.1029/2006GL027494.

\section{Introduction and Background}

[2] The Martian surface is today a cold barren desert. Even during summer in the midlatitudes the ground temperature lies between $230 \mathrm{~K}$ and $180 \mathrm{~K}$ [Carr, 1996]. This coupled with an atmospheric pressure of only $600 \mathrm{~Pa}$ at the reference datum altitude means that surface water is unstable as a liquid. Extensive evidence for the primordial planet being warmer and wetter [Squyres and Kasting, 1994], however, supports the possibility of a Martian genesis of life. Microbes may remain on the surface in cryptoendolith niches [Cockell and Raven, 2004], permafrost water ice [Schulze-Makuch et al., 2005], or active in deep aquifer ecosystems [Boston et al., 1992]. In addition to temperature and liquid water stability, another critical hazard for near-surface life is the radiation environment. This study constitutes the most accurate model to date of the subsurface ionising radiation environment, as a function of both depth and surface composition, and its influence on the survival of different microbes.

[3] Solar UV rapidly deactivates unshielded cells [Cockell and Raven, 2004], and is also believed to have

\footnotetext{
${ }^{1}$ Centre for Mathematics and Physics in the Life Sciences and Experimental Biology (CoMPLEX), University College London, London, UK.

${ }^{2}$ Physikalisches Institut, University of Bern, Bern, Switzerland.

${ }^{3}$ Department of Biochemistry and Molecular Biology, University College London, London, UK.

${ }^{4}$ Mullard Space Science Laboratory, University College London, Dorking, UK.
}

Copyright 2007 by the American Geophysical Union. 0094-8276/07/2006GL027494 created highly oxidising surface conditions that destroy organic molecules in the topsoil. The Viking lander probes found no trace of organic compounds, not even at the level expected from meteoritic infall [Yen et al., 2000]. UV penetrates only millimetres into regolith [Cockell and Raven, 2004], but there is estimated to be around a meter's depth of loose topsoil [Soderblom et al., 2004] within a portion of which wind-mixing of oxidants is expected to present a serious biological hazard. Beyond the penetration depth of UV the ionising radiation of solar energetic protons (SEP) and galactic cosmic rays (GCR) dominate. SEP are accelerated by flares and coronal mass ejections (CME), typically up to several hundred $\mathrm{MeV}$, and so the flux is dependent on the 11-year solar activity cycle. The peak flux of GCR particles, at around $500 \mathrm{MeV} /$ nucleon, is about four orders of magnitude lower than SEP but the power law tail of the spectra extend up to $10^{20} \mathrm{eV}$ at extremely low fluxes. The GCR spectrum is composed of $85 \%$ protons, $14 \%$ alpha, and a small fraction of heavy ions (fully ionised atomic nuclei), and is thought to be mainly accelerated by Type II supernovae. GCR below about $1 \mathrm{GeV} /$ nucleon are modulated by the heliosphere [Klapdor-Kleingrothaus and Zuber, 2000] so their flux is anticorrelated with the solar activity cycle.

[4] SEP are deflected by the geomagnetic field and attenuated by Earth's atmosphere, but the Martian surface is unprotected by a global dipole magnetic field or sufficient atmospheric shielding. The more energetic GCR produce extensive showers of secondary particles in the terrestrial atmospheric column. When a GCR strikes an atmospheric nucleus energetic secondary mesons (pions and kaons), nucleons, gammas and nuclear fragments are produced, which then interact with other nuclei. Secondary mesons decay over a short timescale to produce muons, gamma rays and electrons. Thus the air shower is composed of a central 'hard component' core of nuclear fragments within a spreading 'soft component' cone of the electromagnetic cascade [Eidelman et al., 2004]. The flux of secondaries builds with increasing shielding depth until the Pfotzer maximum, after which the average particle energy is below the threshold for new particle production and the cascade steadily decays. On Earth, this Pfotzer maximum occurs at an altitude of around $15 \mathrm{~km}$; roughly the cruising altitude of Concorde. Similarly-structured cascades occur in the top meters of the Martian regolith.

[5] The ionising radiation field produced by SEP and GCR is harmful to life [Nelson, 2003] through both direct and indirect mechanisms. Direct damage occurs when deposited energy excites electrons within biomolecules, leading to ionisation or radiolysis. However, radiation primarily interacts with water as it comprises $40-70 \%$ of cells and $20 \%$ in bacterial spores, and this creates highly- 
reactive species such as the hydroxyl radical or hydrogen peroxide that then diffuse and attack biomolecules [Baumstark-Khan and Facius, 2001]. The amount of energy deposited by ionising radiation in the target material per unit mass is measured in $\mathrm{J} / \mathrm{kg}$, or Grays (Gy). Bacteria such as E. coli are rapidly inactivated by less than a hundred Gy of gamma radiation, but radioresistant species are known. Gamma-irradiated populations of Deinococcus radiodurans can survive $5 \mathrm{kGy}$ without measurable loss of viability despite massive DNA fragmentation [Cox and Battista, 2005], and $1 \%$ survival is still found after $10 \mathrm{kGy}$. This remarkable resilience is believed to be mainly due to more efficient enzymatic DNA repair and recovery mechanisms [Battista, 1997]. A passive resistance to radiation is exhibited by the spores of Bacillus subtilis. These show no detectable metabolism, but resistance to heat, chemical toxins, gamma radiation and UV is conferred by the largely desiccated interior and a set of small bound proteins protecting the DNA [Setlow, 1994].

[6] Different particles of ionising radiation are not equally hazardous to life. Gamma radiation is weakly ionising, and the energy of a given dose is deposited uniformly throughout the target. The protons and high-charge/high-energy (HZE) ions of the hadronic cascade, however, are highly ionising and deposit energy in a dense track. HZE flux can cause clusters of multiple breaks in DNA strands and is therefore particularly hazardous to cellular survival. The original formulation of absorbed dose does not take into account the dependence of biological effect on the distribution of energy deposition, and various methodologies have arisen to describe the enhanced deleterious effect of HZE particles. This study uses weighting by the relative biological effectiveness (RBE), defined as the dose of a reference radiation (usually gamma or X-rays) required to produce the same biological effect as a dose of the test radiation [Baumstark-Khan and Facius, 2001].

[7] No particle detector has been landed on the Martian surface and so computer modelling is crucial in determining the radiation environment both on the ground and beneath. Work has so far focussed on calculating the surface radiation environment [Molina-Cuberos et al., 2001] and especially the doses absorbed by astronauts during surface exploration or within habitats protected by regolith-derived shielding of variable thickness [Simonsen and Nealy, 1991; Kim et al., 1998; Saganti et al., 2004]. Only limited investigation has yet been performed on the effects to microbial life as a function of depth in the regolith [Mileikowsky et al., 2000; Pavlov et al., 2002]. These aforementioned previous studies use analytic approximations to particle propagation based on the integrodifferential Boltzmann transport equations. Such codes generally necessitate simplifications including target homogeneity, normally incident particles, disregarding the cascade angular spread, and neglecting the generation and propagation of certain secondary particle types (including the extensive showers of gamma, electrons and pions).

\section{Method}

[8] This research employs Geant4 [Agostinelli et al., 2003] to perform a Monte Carlo (MC) simulation of the entire secondary cascades within a full $3 \mathrm{D}$ environment, allowing a precise specification of the geometry, atmospheric and regolithic spatial heterogeneity, reproduction of particle scattering and the actual isotropic angular distribution of incoming primary particles. The Geant 4 physics code performs well in validation tests against experimental data [e.g. Amako et al., 2005; Beringer et al., 2003], with the specific physics description used here from PLANETOCOSMICS (http://cosray.unibe.ch/ laurent/ planetocosmics/). This is the first study to perform such a complete MC simulation of the Martian subsurface radiation environment.

[9] The model comprises a $70 \mathrm{~km}$ atmospheric column atop $20 \mathrm{~m}$ of surface material. The current atmosphere is accurately reproduced in terms of composition, and pressure, density and temperature profiles, with data taken from the Mars Climate Database [http://www-mars.lmd.jussieu. $\mathrm{fr} /]$ to represent noon on a Summer day $(\mathrm{Ls}=270-300)$ within Arabia Terra. This season represents the annual peak for atmospheric density, and the location selected as it lies at the reference altitude with a surface pressure of $600 \mathrm{~Pa}$. Three distinct regolith scenarios were examined. Model (1), 'Dry Homogenous' or DH, is the simplest case and was constructed to allow comparison of results with previous publications. It contains a spatially homogenous $2.8 \mathrm{~g} / \mathrm{cm}^{3}$ block of regolith, devoid of water, and with an elemental composition taken as the mean of Pathfinder analyses of soil samples [Wänke et al., 2001]. Model (2), 'Pure Ice' or PI, is a $1 \mathrm{~g} / \mathrm{cm}^{3}$ block of pure water ice used to emulate environments such as the north polar ice cap, frozen crater lakes or the putative Cerberus pack-ice [Murray et al., 2005]. Model (3), 'Wet Heterogeneous' or WH, was built as a more realistic approximation of the Martian regolith containing permafrost, stratified into a $25 \mathrm{~cm}$ layer of $1.1 \mathrm{~g} / \mathrm{cm}^{3}$ topsoil $2 \%$ water by weight, a $75 \mathrm{~cm}$ thick layer of $1.1 \mathrm{~g} / \mathrm{cm}^{3}$ topsoil with $16 \%$ water, and the bottom $19 \mathrm{~m}$ as $3 \mathrm{~g} / \mathrm{cm}^{3}$ regolith with $16 \%$ water. This layering reproduces the model of subsurface permafrost in Arabia Terra based on neutron backscatter data [Mitrofanov et al., 2004], but is also a good approximation of ice-laden regolith in high latitudes. The elemental compositions of topsoil and regolith strata were taken as the Pathfinder average soil and calculated soil-free rock [Wänke et al., 2001] respectively, although previous studies have found the exact elemental composition to have negligible effect on the shielding properties of regolith [Kim et al., 1998]. These scenarios were chosen for this present study, but the model can be rapidly reconfigured to simulate any atmospheric condition or surface composition, as well as deflection through magnetic fields such as the crustal anomalies.

[10] GCR primary spectra for $Z=1-26$ (protons to iron nuclei) were taken from the CREME96 model [https:// creme96.nrl.navy.mil/] under solar minimum conditions to represent a worse-case scenario for GCR flux, and extrapolated to $1 \mathrm{TeV} /$ nucleon. SEP flux over $10-200 \mathrm{MeV}$ was taken from the SPENVIS database [http://www.spenvis. oma.be/], adjusted to Martian orbit and averaged over an entire 11-year solar activity cycle. Data from $\mathrm{H}, \mathrm{He}$, and $\mathrm{C}$ primaries were weighted by energy integration and combined to model the entire GCR spectra $100 \mathrm{MeV} /$ nuc $-1 \mathrm{TeV} /$ nuc. Particle energy spectra were recorded at the surface and regular intervals though the regolith along with the energy deposited within a micron-thick layer of water to give the 

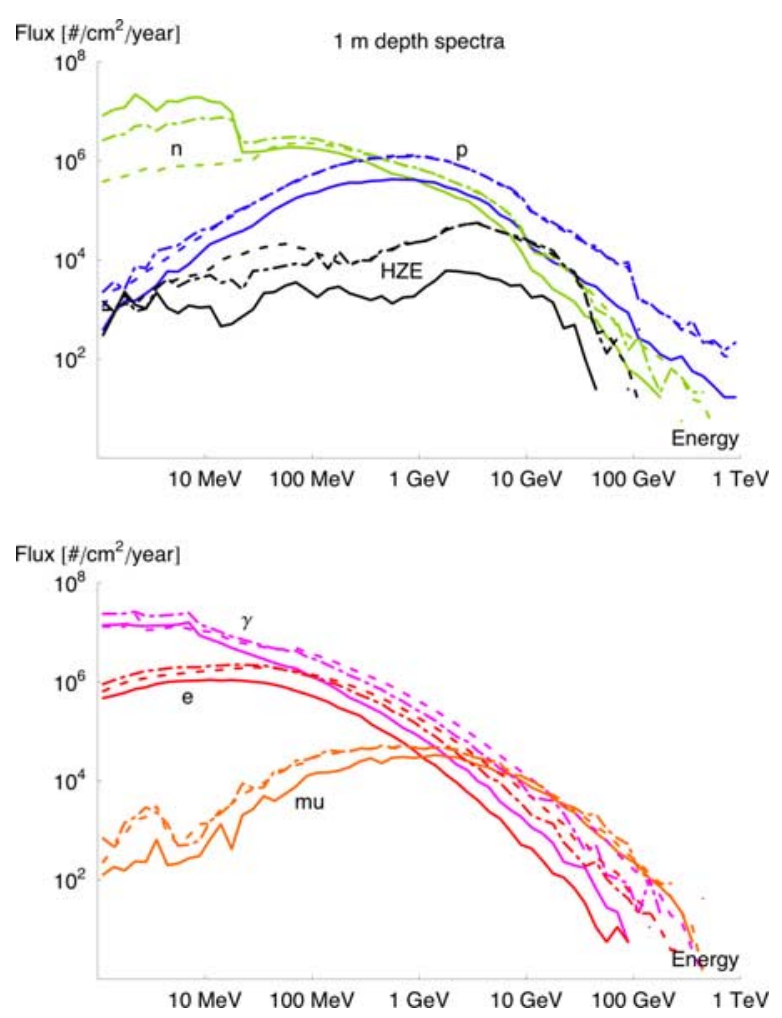

Figure 1. Energy spectra of six most important particles at $1 \mathrm{~m}$ depth. Surface model indicated as follows: DH (solid), PI (dashed), and WH (dot-dash).

microbial absorbed dose, which was also biologically weighted according to particle type and energy using reasonable RBE values [International Commission on Radiological Protection, 1990] as previously demonstrated [Pavlov et al., 2002]. Complete inactivation of a microbial population was taken at the accumulated dose yielding a $10^{-6}$ survival fraction; extrapolated to be $1 \mathrm{kGy}$ and $15 \mathrm{kGy}$ for E. coli and D. radiodurans respectively [Battista, 1997], and $8.4 \mathrm{kGy}$ for B. subtilis spores [Baumstark-Khan and Facius, 2001]. These three organisms are taken as models of non-radioresistance, radioresistance through improved cellular repair and recovery mechanisms, and passive resistance through sporulation, respectively. They are reasonable representatives of possible Martian microbes assuming evolution of a comparable biochemistry or panspermic cross-fertilisation of Mars and Earth, but are also relevant to the issue of planetary protection and survival of humandelivered contaminant cells.

\section{Results}

[11] The surface flux of protons, HZE and muons is invariant on the ground model, as expected since this hard component of the cascade does not backscatter and so differences in regolith composition have no effect on surface flux. The DH model finds $56 \%$ of surface neutrons $<450 \mathrm{MeV}$ are backscattering, however. Figure 1 shows the energy spectra at $1 \mathrm{~m}$ depth across six orders of magnitude $(1 \mathrm{MeV}$ to $1 \mathrm{TeV})$ of the most important particle species, protons, HZE, neutrons, gamma, electrons/positrons and muons, although it should again be noted that the MC methodology employed here generates and tracks all sec- ondary particles in both the hadronic and electromagnetic cascades. The lower energy neutron spectra can be seen to be strongly dependent on the regolith model. This is due to the efficient moderation and capture of neutrons by the high hydrogen content of water. The de-excitation of hydrogen nuclei following thermal neutron capture produces a 2.2 MeV gamma, and this distinctive signal is clearly seen in the PI gamma line in Figure 1. By $1 \mathrm{~m}$ depth, high energy HZE have been strongly absorbed by the regolith, with the lower energies showing least reduction from the primary spectrum due to regeneration by nuclear fragmentation within the hadronic cascade. These energy spectra are not specific to microbial targets and so can also be used to calculate the dose absorbed by astronauts within habitats protected by regolith-derived shielding.

[12] Figure 2 shows the flux of proton, HZE, neutron, muon, gamma and $\mathrm{e}^{-} / \mathrm{e}^{+}$secondaries as a function of depth. Both hadronic and electromagnetic cascades can be seen to be attenuated least by the PI model, due to the lower shielding density of the ice target. Neutron flux, however, is attenuated quickest within the WH regolith as this shielding material is both hydrogenous and dense. Pfotzer maxima are observed within the gamma and electron depthprofiles of all three regolith models, occurring $1 \mathrm{~m}$ deeper in PI than the more dense DH target. In all regolith scenarios the gamma flux declines until it meets the muon profile, with these weakly-interacting secondaries penetrating at appreciable levels to the full $20 \mathrm{~m}$ depth modelled. The electron flux follows at a factor of five lower as they are produced by muon decay and gamma pair-production. On
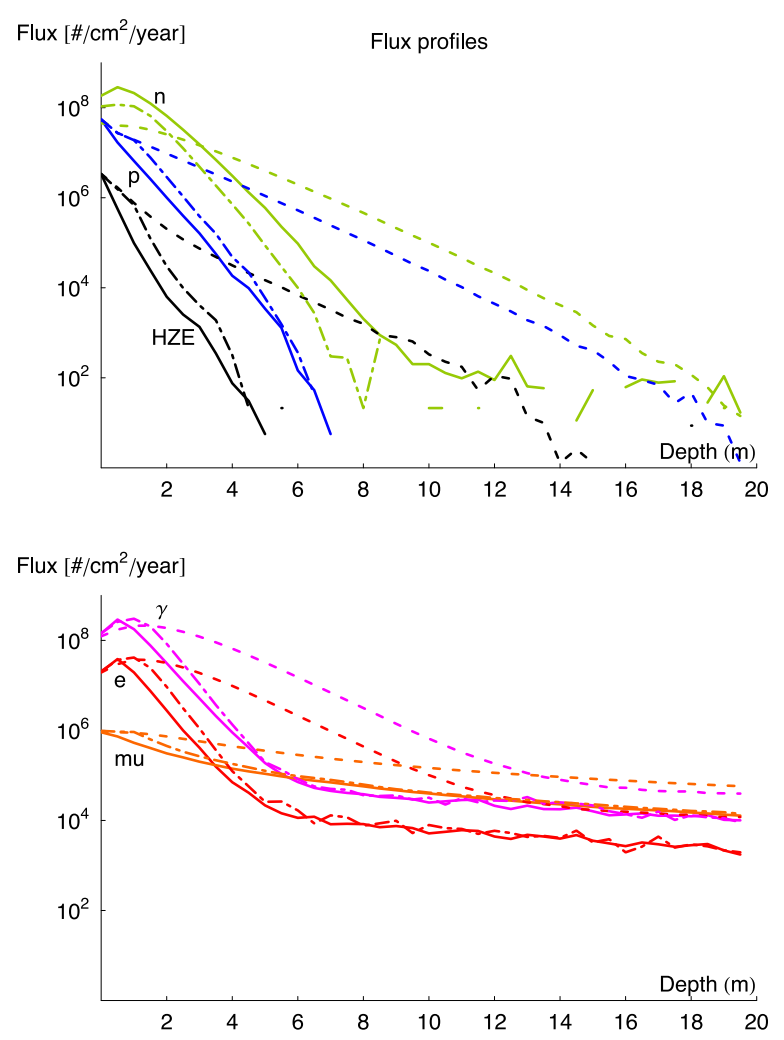

Figure 2. Attenuation with depth for (top) hadronic core, and (bottom) muons and electromagnetic cascade. Surface model indicated as follows: DH (solid), PI (dashed), and WH (dot-dash). 

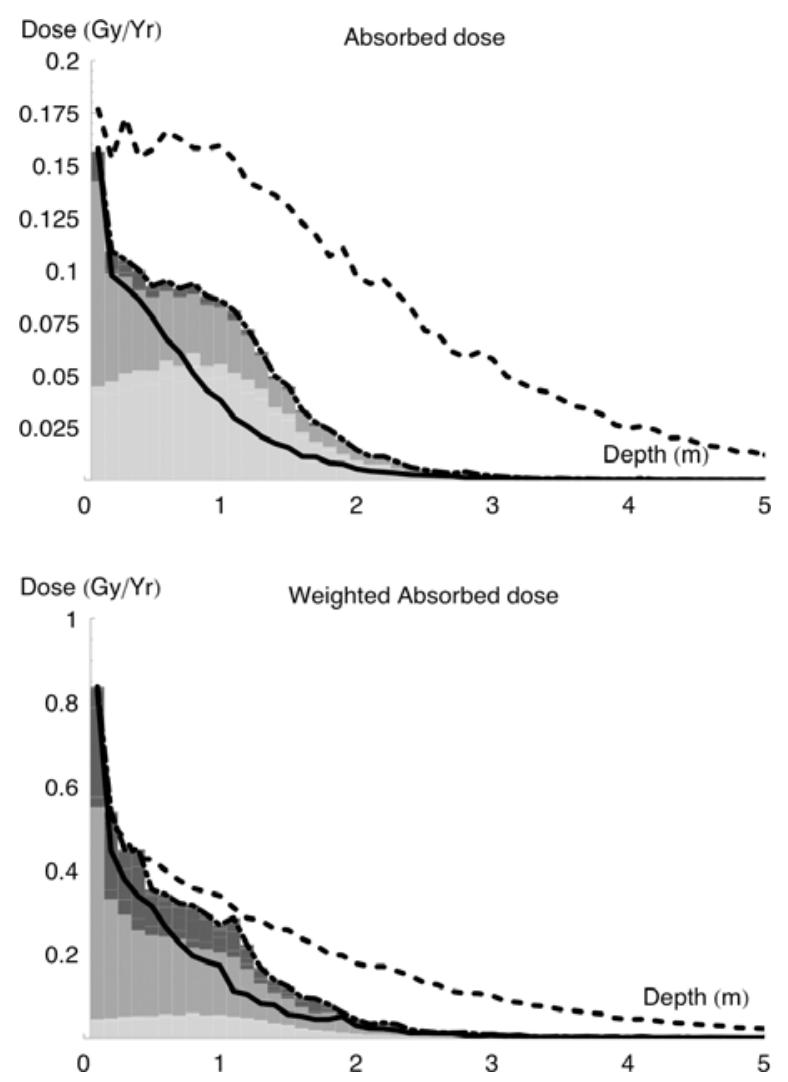

Figure 3. The decline in (top) absorbed dose and (bottom) biologically-weighted dose, with depth. Shown for all three surface models: DH (solid), PI (dashed), and WH (dotdash); and energy deposition within WH model segmented into muon and $\mathrm{e}^{-} / \mathrm{e}^{+}$(light gray), proton (medium gray), and HZE (dark gray).

average, a microbial cell (taken to have cross-sectional area of $\left.10^{-12} \mathrm{~m}^{2}\right)$ on the surface is traversed by an HZE $(Z \geq 2)$ every 30 years, and every 145 years $1 \mathrm{~m}$ deep in WH.

[13] Figure 3 (top) plots the annually-accumulated absorbed dose as a function of depth for all three surface models, and Figure 3 (bottom) the biologically-weighted dose. The surface weighted dose is almost $0.85 \mathrm{~Gy} /$ year for all three models, of which in DH $20 \%$ is mediated by backscattering particles and 35\% contributed from SEP. A single flare can deliver several percent of the average annual fluence in a matter of hours. The proton spectrum recorded from the very hard January 20th 2005 event [Mewaldt et al., 2005] was corrected for Martian orbit and run through the model. The calculated surface dose rate approaches $1 \times$ $10^{-3} \mathrm{~Gy} / \mathrm{hr}$. Although present at a high flux, SEP are not particularly energetic and penetrate less than $10 \mathrm{~cm}$ in the two rocky surfaces and less than $20 \mathrm{~cm}$ in the PI model. A segmented bar chart showing the dose contributions from different particles has been overlain the curve for the WH model. Energy deposition by all particles is included, but HZE, protons, muons and $\mathrm{e}^{-} / \mathrm{e}^{+}$dominate. The absorbed dose graph shows the surface spike from SEP flux, with the proton influence steadily diminishing with depth, and muon and $\mathrm{e}^{-} / \mathrm{e}^{+}$deposition becoming increasingly dominant in the radiation field. Averaged over the top meter of WH regolith HZE particles contribute only $7 \%$ of the absorbed dose, but due to their disproportionately deleterious effect at the cellular level make up $31 \%$ of the weighted absorbed dose as seen in Figure 3 (bottom). Using the annual accumulation of weighted dose it is possible to determine the population survival times (defined above as a million-fold decrease in viable cell number) for each of the three model organisms as a function of depth within each of the surface scenarios, as shown in Table 1.

[14] Verification of these dose-profile results against other studies was performed using the DH regolith scenario and considering only GCR primaries. The surface weighted dose of $0.54 \mathrm{~Gy} /$ year corresponds well with the findings of Mileikowsky et al. [2000] and Pavlov et al. [2002], although as expected is higher due to the inclusion of backscattering and electromagnetic cascade particles.

\section{Discussion and Conclusions}

[15] With a peak biologically-weighted dose rate of $\sim 0.85 \mathrm{~Gy} /$ year at no point is the ionizing radiation environment on or beneath the Martian surface lethal to even radiosensitive terrestrial bacteria. For comparison, the most intense natural radiation environment on Earth is reported to be the monazite sand deposits along certain beaches in Brazil with a dose rate of 0.4 Gy/year [Baumstark-Khan and Facius, 2001], corresponding to a depth of about $20 \mathrm{~cm}$ in the WH regolith. Terrestrial species such as $D$. radiodurans can recover from doses four-orders of magnitude greater than this annual level with no detectable loss in viability [Battista, 1997]. Extreme radioresistance of terrestrial bacteria is therefore not thought to have evolved as an adaptation to the natural radiation environment per se, but as a side-effect of desiccation resistance [Battista, 1997], and there is no reason Martian microbes might not have evolved even greater radioresistance.

[16] Only metabolically active cells, however, are able to repair radiation damage and reproduce. The current freezing conditions in the near subsurface imply that any extant life will be held dormant and it is the dose accumulated over long time periods that will be crucial in determining cell survival. In terms of planetary protection, Table 1 shows that a contaminant population of terrestrial cells or spores deposited onto the Martian surface by a lander probe need only be blown under a thin layer of dust for protection from rapid deactivation by UV and they will survive the SEP and GCR flux for millennia. Over geological time-scales, though, even the most radioresistant populations are inactivated. At $2 \mathrm{~m}$ depth, for example, the ExoMars drill length [Vago et al., 2006], a D. radiodurans population dormant in regolith permafrost suffers a million-fold cull in 450,000 years. For the prospects of finding viable Martian microbes then, cells must either have been brought to the surface only recently, by outflow of deeper meltwater or exposure by impact excavation, for example, or else be able to periodically revive to repair radiation damage and reproduce, and so reset the inactivation clock.

[17] Episodic local geothermal events may melt pockets of near surface permafrost to allow transient bursts of metabolism and replication. Intriguingly, the gullies and seeps seen at high latitude suggest the recent transient presence of liquid water [Malin and Edgett, 2000]. Furthermore, calculations show the near-surface temperature of high latitude ice may be high enough to allow liquid water 
Table 1. Summary of Doses and Microbial Survival Times at Depth

\begin{tabular}{|c|c|c|c|c|c|}
\hline \multirow[b]{2}{*}{ Depth, m } & \multirow{2}{*}{$\begin{array}{l}\text { Surface } \\
\text { Model }^{\mathrm{a}}\end{array}$} & \multirow{2}{*}{$\begin{array}{c}\text { Weighted } \\
\text { Dose, }{ }^{\mathrm{b}} \text { Gy/year }\end{array}$} & \multicolumn{3}{|c|}{ Survival Time,${ }^{\mathrm{c}}$ years } \\
\hline & & & E. coli & B. subtilis & D. radiodurans \\
\hline \multirow[t]{3}{*}{0.0} & DH & 0.83 & 1,200 & 10,100 & 18,100 \\
\hline & PI & 0.83 & 1,200 & 10,100 & 18,100 \\
\hline & WH & 0.84 & 1,200 & 10,000 & 17,900 \\
\hline \multirow[t]{3}{*}{0.5} & DH & 0.27 & 3,800 & 31,700 & 56,600 \\
\hline & PI & 0.40 & 2,500 & 21,000 & 37,500 \\
\hline & WH & 0.34 & 2,900 & 24,500 & 43,800 \\
\hline \multirow[t]{3}{*}{1.0} & DH & 0.11 & 8,900 & 75,000 & 134,000 \\
\hline & PI & 0.31 & 3,200 & 27,000 & 48,000 \\
\hline & WH & 0.29 & 3,500 & 29,000 & 52,000 \\
\hline \multirow{3}{*}{2.0} & $\mathrm{DH}$ & 0.02 & 43,000 & 370,000 & 650,000 \\
\hline & PI & 0.17 & 5,900 & 50,000 & 90,000 \\
\hline & WH & 0.03 & 30,000 & 250,000 & 450,000 \\
\hline \multirow[t]{3}{*}{5.0} & DH & $6.5 \times 10^{-4}$ & $1.5 \times 10^{6}$ & $1.3 \times 10^{7}$ & $2.3 \times 10^{7}$ \\
\hline & PI & $2.5 \times 10^{-2}$ & $4.1 \times 10^{4}$ & $3.4 \times 10^{5}$ & $6.1 \times 10^{5}$ \\
\hline & WH & $2.0 \times 10^{-4}$ & $5.1 \times 10^{6}$ & $4.3 \times 10^{7}$ & $7.6 \times 10^{7}$ \\
\hline \multirow[t]{3}{*}{10.0} & $\mathrm{DH}$ & $2.3 \times 10^{-5}$ & $4.3 \times 10^{7}$ & $3.6 \times 10^{8}$ & $6.5 \times 10^{8}$ \\
\hline & PI & $6.0 \times 10^{-4}$ & $1.7 \times 10^{6}$ & $1.4 \times 10^{7}$ & $2.5 \times 10^{7}$ \\
\hline & WH & $3.4 \times 10^{-5}$ & $3.0 \times 10^{7}$ & $2.5 \times 10^{8}$ & $4.5 \times 10^{8}$ \\
\hline \multirow[t]{3}{*}{20.0} & DH & $6.7 \times 10^{-6}$ & $1.5 \times 10^{8}$ & $1.3 \times 10^{9}$ & $2.2 \times 10^{9}$ \\
\hline & PI & $4.8 \times 10^{-5}$ & $2.1 \times 10^{7}$ & $1.7 \times 10^{8}$ & $3.1 \times 10^{8}$ \\
\hline & WH & $6.8 \times 10^{-6}$ & $1.5 \times 10^{8}$ & $1.2 \times 10^{9}$ & $2.2 \times 10^{9}$ \\
\hline
\end{tabular}

${ }^{\mathrm{a}} \mathrm{DH}$, dry homogenous; PI, pure ice; $\mathrm{WH}$, wet heterogeneous.

${ }^{\mathrm{b}}$ Not including radionuclide contribution. Values shown for depths $>2 \mathrm{~m}$ are averaged over $\pm 0.5 \mathrm{~m}$.

${ }^{\mathrm{c}} 10^{-6}$ population reduction.

and thus metabolism during periods of high obliquity [Jakosky et al., 2003]. However, liquid water is predicted during these times only in the top meter of ice, and at this depth populations of even radioresistant bacteria are inactivated in the order of tens of thousands of years, far short of the calculated obliquity shift frequency on the order of tens of millions of years [Laskar and Robutel, 1993].

[18] One site of particular astrobiological interest is the putative frozen sea observed on the plains of Elysium [Murray et al., 2005]. This is believed to have been disgorged by the Cerberus Fossae only 5 million years ago [Murray et al., 2005], rapidly freezing in the current climate, and presents an opportunity for our landers to sample water from much greater depths than they could otherwise access. Assuming cellular dormancy since soon after this discharge event, the PI model predicts a bore hole of around $7.5 \mathrm{~m}$ would be needed for any hope of retrieving culturable $D$. radiodurans-like bacteria.

[19] Ionizing radiation is also produced by radionuclide decay, and it has been calculated that the intrinsic radioactivity of Martian regolith produces a dose of $4 \times$ $10^{-4}$ Gy/year [Mileikowsky et al., 2000]. Within the WH regolith model this corresponds to a depth of $\sim 4.5 \mathrm{~m}$ at which the background activity begins to dominate over the GCR cascade. Thus beneath this depth dormant cells receive no further shielding benefit, and $D$. radiodurans can be inactivated by radioactivity of the regolith alone in under 40 million years. The near-zero radionuclide content of pure ice implies that frozen crater lakes or the polar caps are the most favourable environments for finding viable cells after long periods of dormancy.

[20] Acknowledgments. L.R. Dartnell is supported by an EPSRC Doctoral Training Centre studentship. L. Desorgher is supported by the Swiss National Science Foundation, grant 200020-105435/1, and by the Swiss State Secretariat for Education and Research, SER, grant COST-724/ C05.0034.

\section{References}

Agostinelli, S., et al. (2003), GEANT4-A simulation toolkit, Nucl. Instrum. Methods Phys. Res., Sect. A, 506, 250-303.

Amako, K., et al. (2005), Comparison of Geant4 electromagnetic physics models against the NIST reference data, IEEE Trans. Nucl. Sci., 52, $910-$ 918, doi:10.1109/TNS.2005.852691.

Battista, J. R. (1997), Against all odds: The survival strategies of Deinococcus radiodurans, Annu. Rev. Microbiol., 51, 203-224, doi:10.1146/ annurev.micro.51.1.203.

Baumstark-Khan, C., and R. Facius (2001), Life under conditions of ionizing radiation, in Astrobiology: The Quest for the Conditions of Life, edited by G. Horneck and C. Baumstark-Khan, pp. 260-283, Springer, New York.

Beringer, J., G. Folger, F. Gianotti, A. Ribon, J. P. Wellisch, D. Barberis, M. Cervetto, and B. Osculati (2003), Validation of Geant4 hadronic physics, in 2003 IEEE Nuclear Science Symposium Conference Record, pp. 494-498, doi:10.1109/NSSMIC.2003.1352091, IEEE Press, Piscataway, N. J.

Boston, P. J., M. V. Ivanov, and C. P. McKay (1992), On the possibility of chemosynthetic ecosystems in subsurface habitats on Mars, Icarus, 95, 300-308, doi:10.1016/0019-1035(92)90045-9.

Carr, M. H. (1996), Water on Mars, 229 pp., Oxford Univ. Press, New York.

Cockell, C. S., and J. A. Raven (2004), Zones of photosynthetic potential on Mars and the early Earth, Icarus, 169, 300-310, doi:10.1016/ j.icarus.2003.12.024.

Cox, M. M., and J. R. Battista (2005), Deinococcus radiodurans-The consummate survivor, Nature Rev. Microbiol., 3, 882-892, doi:10.1038/nrmicro1264.

Eidelman, S., et al. (2004), Review of particle physics, Phys. Lett. B, 592, $1-5$, doi:10.1016/j.physletb.2004.06.001.

International Commission on Radiological Protection (1991), 1990 Recommendations of the International Commission on Radiological Protection, Annals of the ICRP, ICRP Publ., vol. 60, Elsevier, New York.

Jakosky, B. M., K. H. Nealson, C. Bakermans, R. E. Ley, and M. T. Mellon (2003), Subfreezing activity of microorganisms and the potential habitability of Mars' polar regions, Astrobiology, 3, 343-350, doi:10.1089/ 153110703769016433.

Kim, M.-H. Y., S. A. Thibeault, L. C. Simonsen, and J. W. Wilson (1998), Comparison of Martian meteorites and Martian regolith as shield materials for galactic cosmic rays, NASA Tech. Pap., NASA TP-1998-208724.

Klapdor-Kleingrothaus, H. V., and K. Zuber (2000), Cosmic radiation, in Particle Astrophysics, chap. 8, pp. 223 -247, Inst. of Phys., Bristol, U. K.

Laskar, J., and P. Robutel (1993), The chaotic obliquity of the planets, Nature, 361, 608-612, doi:10.1038/361608a0.

Malin, M. C., and K. S. Edgett (2000), Evidence for recent groundwater seepage and surface runoff on Mars, Science, 288, 2330-2335, doi:10.1126/science.288.5475.233. 
Mewaldt, R. A., M. D. Looper, C. M. S. Cohen, G. M. Mason, D. K. Haggerty, M. I. Desai, A. W. Labrador, R. A. Leske, and J. E. Mazur (2005), Solar-particle energy spectra during the large events of OctoberNovember 2003 and January 2005, paper presented at 29th International Cosmic Ray Conference, Tata Inst. of Fundam. Res., Pune, India, 3-10 Aug.

Mileikowsky, C., et al. (2000), Natural transfer of viable microbes in space. 1. From Mars to Earth and Earth to Mars, Icarus, 145, 391-427.

Mitrofanov, I. G., et al. (2004), Soil water content on Mars as estimated from neutron measurements by the HEND instrument onboard the 2001 Mars Odyssey spacecraft, Sol. Syst. Res., 38, 253-257, doi:10.1023/ B:SOLS.0000037461.70809.45.

Molina-Cuberos, G. J., W. Stumptner, H. Lammer, N. I. Kömle, and K. O'Brien (2001), Cosmic ray and UV radiation models on the ancient Martian surface, Icarus, 154, 216-222, doi:10.1006/icar.2001.6658.

Murray, J. B., et al. (2005), Evidence from the Mars Express High Resolution Stereo Camera for a frozen sea close to Mars' equator, Nature, 434, 352-356, doi: 10.1038 /nature03379.

Nelson, G. A. (2003), Fundamental space radiobiology, Gravitational Space Biol. Bull., 16, 29-36.

Pavlov, A. K., A. V. Blinov, and A. N. Konstantinov (2002), Sterilization of Martian surface by cosmic radiation, Planet. Space Sci., 50, 669-673, doi:10.1016/S0032-0633(01)00113-1.

Saganti, P. B., F. A. Cucinotta, J. W. Wilson, L. C. Simonsen, and C. Zeitlin (2004), Radiation climate map for analyzing risks to astronauts on the Mars surface from galactic cosmic rays, Space Sci. Rev., 110, 143-156, doi:10.1023/B:SPAC.0000021010.20082.1a.

Schulze-Makuch, D., L. N. Irwin, J. H. Lipps, D. LeMone, J. M. Dohm, and A. G. Fairén (2005), Scenarios for the evolution of life on Mars, J. Geophys. Res., 110, E12S23, doi:10.1029/2005JE002430.
Setlow, P. (1994), Mechanisms which contribute to the long-term survival of spores of Bacillus species, Soc. Appl. Bacteriol. Symp. Ser., 23, 49S$60 \mathrm{~S}$.

Simonsen, L. C., and J. E. Nealy (1991), Radiation protection for human missions to the Moon and Mars, NASA Tech. Pap., NASA TP-3079.

Soderblom, L. A., et al. (2004), Soils of Eagle Crater and Meridiani Planum at the Opportunity Rover landing site, Science, 306, 1723-1726, doi:10.1126/science.1105127.

Squyres, S. W., and J. F. Kasting (1994), Early Mars: How warm and how wet?, Science, 265, 744-749.

Vago, J., B. Gardini, G. Kminek, P. Baglioni, G. Gianfiglio, A. Santovincenzo, S. Bayón, and M. van Winnendael (2006), ExoMars: Searching for life on the red planet, ESA Bull., 17-23.

Wänke, H., J. Bruckner, G. Dreibus, R. Rieder, and I. Ryabchikov (2001), Chemical composition of rocks and soils at the Pathfinder site, Space Sci. Rev., 96, 317-330, doi:10.1023/A:1011961725645.

Yen, A. S., S. S. Kim, M. H. Hecht, M. S. Frant, and B. Murray (2000), Evidence that the reactivity of the Martian soil is due to superoxide ions, Science, 289, 1909-1912, doi:10.1126/science.289.5486.1909.

A. J. Coates, Mullard Space Science Laboratory, University College London, Holmbury St. Mary, Dorking RH5 6NT, UK.

L. R. Dartnell, Centre for Mathematics and Physics in the Life Sciences and Experimental Biology, University College London, Wolfson House, 4 Stephenson Way, London NW1 2HE, UK. (1.dartnell@ucl.ac.uk)

L. Desorgher, Physikalisches Institut, University of Bern, Sidlerstrasse 5 , CH-3012 Bern, Switzerland.

J. M. Ward, Department of Biochemistry and Molecular Biology, University College London, Gower Street, London WC1E 6BT, UK. 